\title{
Spectrum Window Based Signal Detection at Low SNR
}

\author{
Harri Saarnisaari \\ Centre for Wireless Communications \\ University of Oulu \\ Oulu, Finland \\ harri.saarnisaari@oulu.fi
}

\author{
Johanna Vartiainen \\ Centre for Wireless Communications \\ University of Oulu \\ Oulu, Finland \\ johanna.vartiainen@oulu.fi
}

\begin{abstract}
Signal detection is of interest in spectrum awareness that is useful for future cognitive military radios for their spectrum usage decisions. Detection is often based on energy on a narrow bandwidth or frequency bin. For wideband signals covering several frequency bins a spectrum matching detector has been proved to be more sensitive. This requires correlation with several a priori known candidate spectra making it more complicated than the single bin detector. Herein, a simplified version of spectrum matching detector using a rectangular reference spectrum is investigated. It is shown that the proposed simplified version outperforms the single bin version. It can detect wideband signals even below noise level. Furthermore, it was observed that there are several issues that should be solved before the detector could be used in practice. One is that many rectangular window sizes can detect the same signal and, as a consequence, one should find a method to decide which one is the correct one.
\end{abstract}

Index Terms - signal detection, windowing, overlapping

\section{INTRODUCTION}

Situation awareness is a key tool for success in military world. Future military cognitive radio systems could benefit of spectrum situational awareness. since they can adapt their signal and spectrum usage based on spectrum information. A base line spectrum information could be that there are signals present at these frequencies but better yet, there should be some indications what signals they are since then reactions could be better designed.

Spectrum analyzers, frequently used for detecting signals, calculate signal energy in a rather narrow (but adjustable) frequency band. When these outputs are put side-by-side, the spectrum of the total analyzed bandwidth can be shown. Detection is based on a detection threshold and if the energy in a narrow frequency band (called frequency bin or bin hereafter) exceeds the threshold it is declared that there was a signal present at that bin. However, this does not tell anything about the bandwidth of the signal that can be used for signal characterization.

An attempt to estimate the bandwidth was made in [1] and [2]. Therein, a method that analyzes how many adjacent bins contain a signal can be found. Furthermore, it can detect if there are multiple signals even close by. The method is called the localization algorithm based on double-thresholding (LAD) method. It is based on a constant false alarm (CFAR) technique called forward consecutive mean excision (FCME) [3] [4] that sets the detection threshold without a priori information about the noise level. However, the detection in the LAD method is based on an energy in a single bin that is not necessarily optimal if the signal bandwidth is larger than that.

A better approach is to utilize known signal spectra in detection. Herein, a priori known signal spectrum is used to correlate the calculated spectrum to find a signal with that bandwidth. This technique is called the spectrum matching detection technique. This techniques was applied probably first applied in material science [5] and then later in signal detection [6], [7] and [8]. This method requires correlation with respect all (or desired) a priori known spectra. An example of this is described in [9], which describes a product unfortunately (but understandable) without technical details of the method itself. This multicorrelation property means that the method may become rather complicated. In addition, it is not clear how well similar (in bandwidth but not spectrum) signals could be separated by this technique.

An attempt to simplify the spectrum matching technique is proposed here. Multiple rectangular spectrum shapes are used instead of specially matched spectra. The rectangular window bandwidths are based on a priori information about possible signals. Furthermore, the use of FCME technique to set the detection threshold with the proposed spectrum window method is considered. The proposed method's performance is compared to that of the LAD method that could be seen as a benchmark in the class of single bin based detection techniques. The rest of the paper is organized as follows. The next sections briefly describe the proposed method and the reference method. Thereafter, the signal detection threshold setting is discussed followed by numerical results. Finally, conclusions and future ideas around the proposed spectrum window based signal detection are provided.

\section{Window Based Spectrum Matching Method}

In the original spectrum matching method the observed spectrum $S(f)$ is correlated by candidate spectra $C_{i}(f)$. The decision statistics are the outputs of these corrections. Herein, the candidate spectra are rectangular windows. This means that correlation operation reduces to pure (energy) addition 


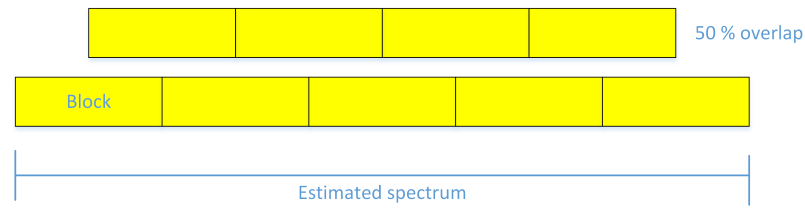

Fig. 1. An illustration of dividing the spectrum in to blocks (50\% overlap example).

operation that simplifies the process somewhat and the decision statistics are the added energy inside a window. It is possible to use the sliding window approach for calculating the correlation. However, it is not clear how much difference there would be in decision statistics if the window contents differ only by one frequency bin. Therefore, the overlapping windows approach, where the overlap could be defined, might be a better solution.

There are $N$ frequency bins $Y_{i}$ computed by some spectrum (power spectral density) estimation method. The bins are divided into $L$ overlapping blocks $Y_{i}(l) \in\left\{Z_{l}\right\}$ with length $M$. Overlap could vary from zero (no overlap) to $M-1$ (sliding window). This is illustrated in Fig. 1.

The decision variables are the total energy of $E_{l}$ of each block, i.e., the sum of elements in the block. The energies are compared to a detection threshold $T_{h}$ and if the energy exceeds the threshold it is declared that a signal (with this particular bandwidth) is present and otherwise, a signal is not present. In other worlds, the decision rule could be written as

$$
E_{l}=\sum_{i} Y_{i}(l)>T_{h}
$$

Ideally, the threshold is based on noise only samples. However, their availability in the group of received samples cannot be guaranteed (known). Therefore, CFAR methods where noise only samples are searched have been proposed. The mentioned FMCE method is such a method and threshold setting with it will be discussed in the next section. In order to use that here just replace $Y_{i}$ by $Z_{i}$. This is called the (FCME) enhanced window based method. The "original" one uses all the samples $Z_{i}$ (their sum indeed) to set the threshold, that works fine if other blocks are without signal.

Another problem so far not discussed in the literature around the spectrum matching method is that different spectra (or windows) may cause threshold crossing for the same signal. Ideally, the correct (or best matching) spectrum would provide the highest total score (or metric) but in noisy environment this is not sure. And wider candidate spectrum also cumulate noise energy that still reduces separation capability.

The reason why spectral matching is so interesting could be explained by a simple example. Assume that signal bandwidth is one hundred bins and that it is tried to be detected by one, four, ten and hundred bins detector. The results are shown in Fig. 2. It is very clear that the optimum window is much more sensitive than the ubiquitous single bin detector. Furthermore, even non-optimal four and ten bin detectors are better (more sensitive) than the single bin detector. Naturally, this example

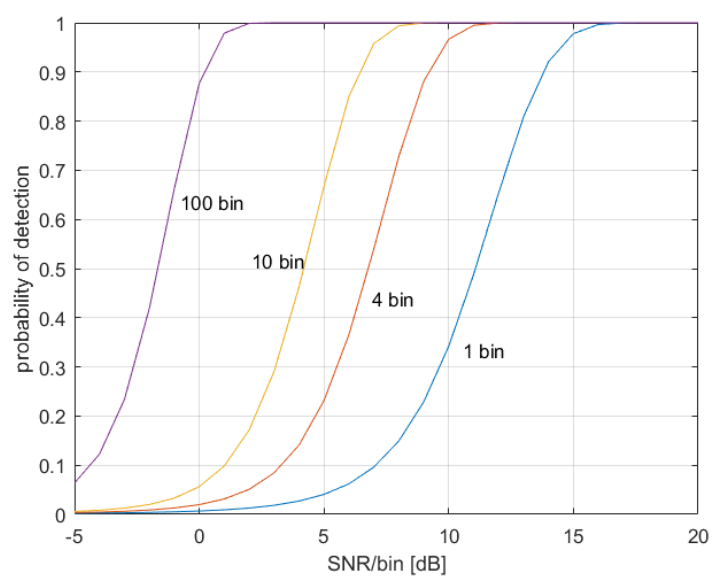

Fig. 2. Comparison of one, four, ten and hundred bin detector for detecting a signal whose bandwidth is one hundred bins and spectrum rectangular.

is oversimplified in the sense that all bins have equal signal energy, which is rarely true in practice, and the threshold is ideally set (base on false alarm rate $10^{-3}$ ). But, still the results show the potential of the spectrum matching method though it makes the detector more complicated. The results are obtained by knowing that in white Gaussian noise bins follow chi-square distribution with $2 M$ degrees-of-freedom.

\section{THE LAD METHOD}

First, the threshold setting principle behind the LAD method is discussed. Variables $Y_{i}$ are arranged in an ascending order according their energy and $x \%$ of the smallest samples form an initial set $Q$. The FCME threshold is [3]

$$
T_{h}=\gamma \frac{1}{Q} \sum_{i=1}^{Q} Y_{i},
$$

where $\gamma$ is based on the desired false alarm rate to be described shortly. The part after $\gamma$ is the estimate of the average noise power. The samples below the threshold are added to the set $Q$, and this iterative process continues until there are no samples below the threshold.

The LAD method [1], [2] uses two of these thresholds, the upper and lower one. The LAD method uses clustering to group adjacent samples assumed to be from the same signal. The LAD method clusters together adjacent samples above the lower threshold. The cluster is accepted to be caused from a signal if at least one of the samples is also above the upper threshold. The performance of the LAD method is improved using an ACC parameter that allows $p$ samples to be below the lower threshold between two accepted clusters [2].

In Fig. 3, there is one snapshot when there is one signal with $10 \%$ bandwidth (of the total bandwidth). In this case, the LAD method separates the signal and finds 3 signals, as the window based method finds one signal. The enhanced window based method finds two signals, at which one is right and one (very narrow) is a falsely detected signal. 


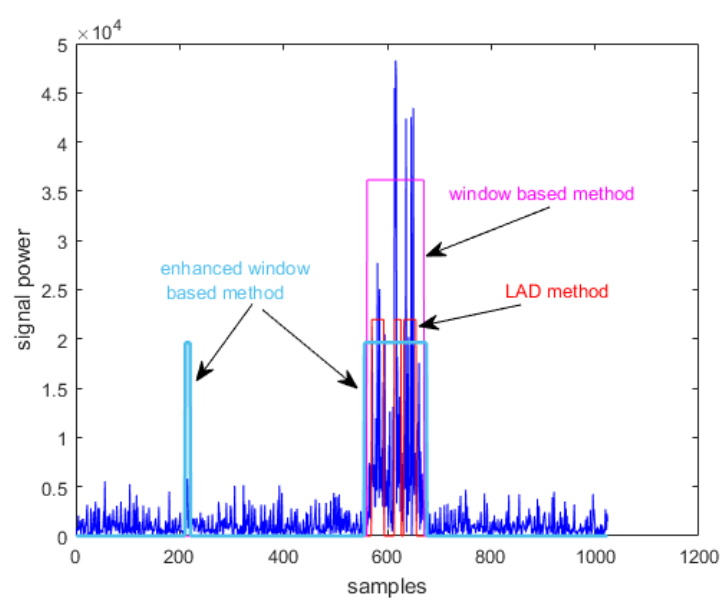

Fig. 3. One snapshot about one simulated signal with $10 \%$ bandwidth. Detected signals using the LAD method, window based method and enhanced window based method.

\section{Threshold Setting}

Setting of $\gamma$ is based on the desired false alarm rate. If the noise is assumed to be a white Gaussian process, noise only samples $Z_{l}$ follow the central chi-squared distribution with $2 M$ degrees of freedom [10]. As a consequence, the threshold parameter $\gamma$ is found by solving

$$
P_{F A}=e^{-\gamma M} \sum_{k=0}^{M-1} \frac{1}{k !}(\gamma M)^{k},
$$

where $P_{F A}$ is a desired false alarm rate.

When $M=1$, variables follow central chi-squared distribution with two degrees of freedom, and (3) comes

$$
\gamma=-\ln P_{F A}
$$

Some threshold parameter values $\gamma$ at different values of $P_{F A}$ and $M$ are presented at Table I.

TABLE I

THRESHOLD PARAMETER VALUES $\gamma$ AT DIFFERENT VALUES OF FALSE ALARM RATE $P_{F A}$ AND $M$.

\begin{tabular}{l|l|l|l|l}
$P_{F A}$ & $M=1$ & $M=4$ & $M=10$ & $M=100$ \\
\hline 0.01 & 2.303 & 1.670 & 1.512 & 1.130 \\
0.001 & 4.605 & 2.511 & 1.878 & 1.247 \\
0.0001 & 6.908 & 3.266 & 2.266 & 1.338
\end{tabular}

\section{Simulation Results}

In the simulations, the window based method and the enhanced window based method were studied and compared with the LAD method. An adjacent version of the LAD method with ACC parameter $p=3$ was used and the LAD threshold parameters were 13.81 and 2.66 , which are typical selections [2]. There is a binary phase shift keying (BPSK) signal with random data buried in complex white Gaussian noise. The signal bandwidth is either $5 \%$ or $10 \%$ of the total bandwidth and the signal is bandlimited by a root raised cosine filter with

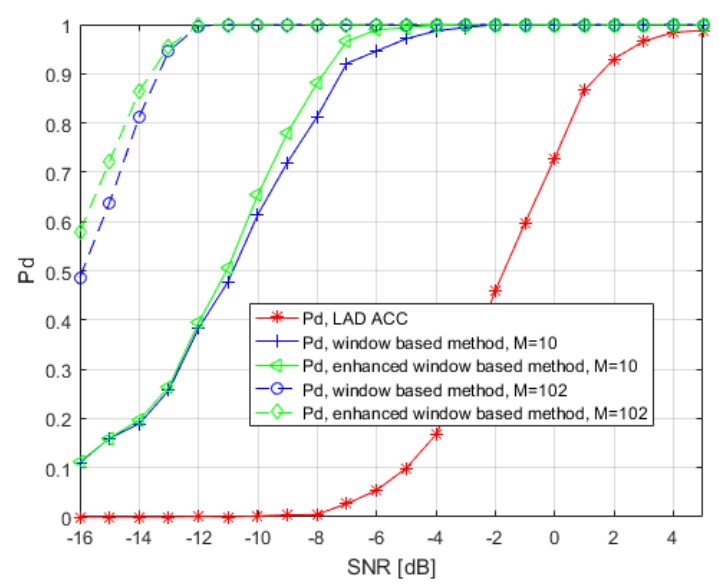

Fig. 4. Probability of detection vs. SNR. The signal bandwidth is $10 \%$ (= 102 samples).

a roll-of factor of 0.22 . The number of samples was $N=1024$, the length of the detection window $(M)$ varied and $50 \%$ overlap was used. The both window based method used false alarm rate 0.01 unless otherwise stated. The probability of detection was defined so that the signal is detected if its correct center frequency is detected. Signal-to-noise ratio (SNR) is defined as total signal power per total noise power (i.e., over 1024 samples). One thousand Monte Carlo iterations were performed. In addition to the probability of detection also the number of detected signals was considered. Especially, this may occur if the window is smaller than the signal bandwidth.

First, the bandwidth of the signal is $10 \%$ or approximately 102 samples. The used window sizes are one (LAD), ten and 102. The detection probability and the number of detected signals are shown in Figs. 4 and 5, respectively. It can be seen that the correct window size is most sensitive, as expected. The shorter window is also more sensitive than the LAD, as expected. Both the LAD and ten bin window tend to overestimate the number of signals when SNR is close to their sensitivity limit, but do better (correctly)when SNR is larger than than. The correct window size gives correct estimate for the number of signals most of the time if it can detect it; it does not overestimate most of the time.

In the second and final case the bandwidth of the signal is $5 \%$, i.e., 51 samples. The window sizes are now one (LAD), four, ten, 52 and 102 meaning that there are both too short and too long windows. The results are shown in Figs. 6 and 7. It can be seen that all windows are more sensitive than the LAD method. The best performance is returned by the correct window (52) and the largest window (102), whose detection performance are almost the same. This despite the fact that the largest window $50 \%$ noise-only samples. Here it can be observed very clearly that several windows can detect the same signal and at the end one has to decide what was correct one. Yes, some windows are more sensitive than others for a particular signal but if SNR is at the correct region this could occur. Conclusions about the number of signals estimation 


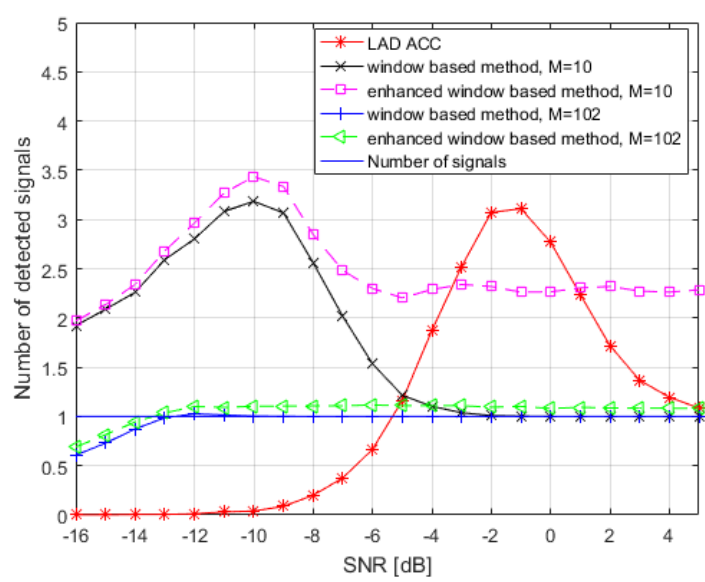

Fig. 5. The number of detected signals vs. SNR. The signal bandwidth is $10 \%$ (=102 samples).

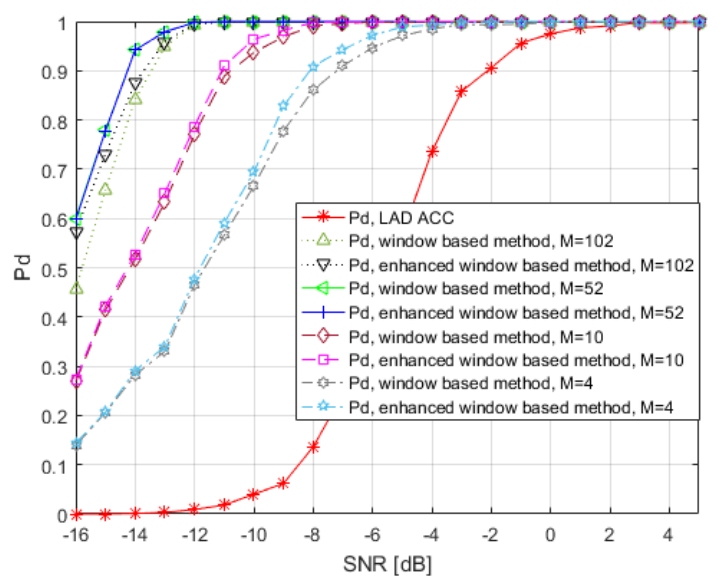

Fig. 6. Probability of detection vs. SNR. Bandwidth of the signal is $5 \%$ (=51 samples).

remains the same except that too large window (102) can also return the correct number of signals most of the time.

It can be concluded that both the window based method outperformed the LAD method and gave a good detection performance even at low SNR values. The enhanced window based detection method gave slightly better detection performance than the window based method but difference was not large. In addition, it was observed that the length of the detection window does not need to match the bandwidth of the detected signal such that the window method is better than the single bin method. Indeed, the window could be shorter or longer (in bandwidth).

When the window equals to that of the signal bandwidth or was larger, the window method found one signal. However, when the window was smaller than the signal bandwidth the methods found too many signals at low SNR values that are close to the sensitivity limits.

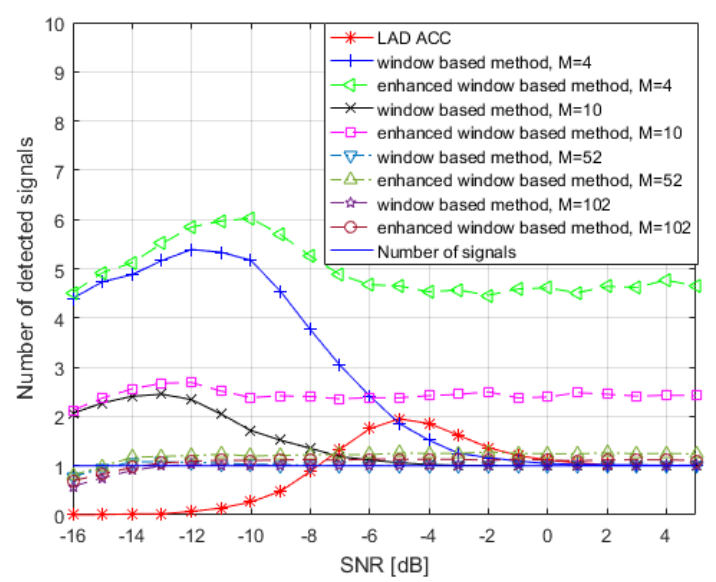

Fig. 7. Number of detected signals vs. SNR. Bandwidth of the signal is $5 \%$ (=51 samples).

\section{CONCLusions}

A spectrum matching based rectangular window based signal detection method was proposed. Its detection threshold setting was discussed and the FCME based approach was proposed. It was shown that the window based detection is much more sensitive for wideband signals whose bandwidth is covering more than one frequency band than single bin detectors, of which the LAD method was used here as a benchmark. However, it was observed that there are still many open issues that should be addressed in further studies.

Herein, the FCME principle was directly applied for threshold setting based on the desired false alarm rate related to the window size but not the total sample size. It is a working approach but is was not checked if its false alarm rate equals the desired one (over all samples). Therefore, the threshold setting should be considered once more by calculating the false alarm probability that depends on the total number of samples.

Then there are a bunch of problems related or depending each other. One question is what is optimal overlap versus complexity and sensitivity? Another question is how many different window lengths are needed again versus complexity and performance? Maybe one could use short (one bin), middle and large sized windows for initial detection and then do more percise analysis for found signals.

In addition, what is the performance difference between rectangular and true spectrum matching; is there significant difference? If the difference in recognizing what was the correct signal/bandwidth is not significant one should use the simplest form.

Furthermore, since many windows can detect the same signal a question is how to select which window is the correct one? How well the used metric works (i.e., energy) performs in this separation or are other metrics needed for this process? Finally, how the previous can be utilized to avoid false detection by too short windows? What matters at the end (as an output of the decision process) is how many and what 
kind of signals were detected, not the intermediate analysis with several windows/spectrum shapes.

\section{REFERENCES}

[1] J. Vartiainen, J. J. Lehtomaki, and H. Saarnisaari, , "Double-threshold based narrowband signal extraction", In IEEE Veh. Technol. Conf, pp. $1288-1292,2005$

[2] J. Vartiainen, "Concentrated signal extraction using consecutive mean excision algorithms". Ph.D. Dissertation, Acta Univ Oul Technica C 368. Faculty of Technology, University of Oulu, Finland, November 2010.

[3] H. Saarnisaari and P. Henttu, "Impulse detection and rejection methods for radio systems", In Mil. Commun. Conf. (MILCOM), pp. 1126-1131, 2003.

[4] H. Saarnisaari, P. Henttu, and M. Juntti, ”Iterative multidimensional impulse detectors for communications based on the classical diagnostic methods", In IEEE Trans. on Communications, vol. 53, pp. 395-398, 2005.

[5] G. R. Hunt, "Rapid remote sensing by a spectrum matching technique: 1. Description and discussion of the method," in J. of Geophysical Research, vol. 71, pp. 2919-2930, June 1966.

[6] H. C. So, W. K. Ma and Y. T. Chan, "Detection of random signals via spectrum matching," in IEEE Trans. on Aerospace and Electronic Systems, vol. 28, Issue 1, January 2002.

[7] Z. Quan, W. Zhang, S. J. Shellhammer and A. H. Sayed,"Optimal Spectral Feature Detection for Spectrum Sensing at Very Low SNR", in IEEE Trans. on Commun., vol. 59, no 1, January 2011, pp.201-212.

[8] E. Lagunas and M. Najar, "Compressive Spectrum Sensing Based on Spectral Shape Feature Detection", in The Tenth International Symposium on Wireless Communication Systems, 2013.

[9] Y. Phuan," The need for speed: Spectral shape detector accelerates signal search", in Rohde\&Schwarz News Magazine, pp. 56-61, 210/14.

[10] J. G. Proakis, "Digital Communications". Third Edition. McGraw-Hill, Inc., 1995. 